\title{
Eicosapentaenoic acid reduces inflammation and apoptosis by SREBP1/TLR4/MYD88
}

\author{
Zhang $\mathrm{L}^{1}$, Jia $\mathrm{NN}^{2}$, Yang $\mathrm{RH}^{1}$, Wang $\mathrm{F}^{1}$ \\ Department of Health Education and Management, School of Military Preventive Medicine, Air Force Medical \\ University, Fourth Military Medical University, Xi'an, Shannxi, China. wangfengsxaf@163.com.
}

\begin{abstract}
AIM: Podocytes dysfunction including the cell integrity, apoptosis and inflammation plays crucial role in diabetic nephropathy. Current exploration evaluated the protective role of eicosapentaenoic acid (EPA) in high glucose-treated podocytes and the underlying mechanisms.

METHOD: MPC5 cell were stimulated by high glucose or treated by EPA of different concentrations. CCK8

assay was utilized to assess MPC5 cell viability, flow cytometry analyzed cell apoptosis.

RESULTS: Data showed that EPA prominently alleviated the high glucose-induced apoptosis and

inflammation. Besides, the disruption of the podocytes structure certifying by podocin and synaptopodin induced by hyperglycemia was hindered by EPA administration. In addition, overexpression of the sterol regulatory element-binding protein-1 (SREBP-1) reversed the protective effects of EPA in high glucose-treated podocytes. EPA inhibits the SREBP-1/TLR4/MYD88 signaling in high glucose treated cells.

CONCLUSIONS: This study suggests that EPA protects against podocytes dysfunction by regulating

SREBP-1 and these findings provide a better understanding for diabetic nephropathy and a novel therapeutic strategy (Fig. 7, Ref. 24). Text in PDF www.elis.sk

KEY WORDS: eicosapentaenoic acid, inflammation, apoptosis, SREBP1/TLR4/MYD88.
\end{abstract}

\section{Introduction}

Diabetic nephropathy (DN) is one of the most common complications of diabetes. The classical management by angiotensin-converting enzyme inhibitors and ARB, angiotensin receptor blocker in diabetes therapy is still connected with residual risk of DN (1). Thus, it is urgent for researchers to exploit new therapeutic options for DN therapy.

The clinical features of DN are the increasing levels of albuminuria and the reduction of glomerular filtration rate (GFR) $(2,3)$. The glomerular endothelium, the basement membrane and podocyte maintain the integrity of the glomerular filtration barrier, the function of which is correlated with the pathophysiology of DN $(4,5)$. Recently, targeting glomerular hyperfiltration, inflammation, and fibrosis are considered novel therapeutic strategies (1). Dysfunction of podocytes leads to albuminuria, glomerular hypertrophy and glomerular basement membrane thickness and finally chronic renal failure (6). The podocytes injury mainly includes extra cel-

\footnotetext{
${ }^{1}$ Department of Health Education and Management, School of Military Preventive Medicine, Air Force Medical University, Fourth Military Medical University, Xi'an, Shannxi, China, ${ }^{2}$ Department of Clinical Immunology, Xijing Hospital, Air Force Medical University, Fourth Military Medical University, Xi'an, Shannxi, China

Address for correspondence: F. Wang, Prof, Department of Health Education and Management, School of Military Preventive Medicine, Air Force Medical University, Fourth Military Medical University, 169 Changle West Road, Xi'an, Shannxi, 710032, China.

Phone: +86.029 .84774483$
}

lular matrix dysfunction, abnormal gene expression (e.g. nephrin, podocin, and cadherin), aberrant actin cytoskeleton and podocytes apoptosis. Inflammation, as well as oxidative stress and fibrosis are key regulators for initiation of DN (7). Inhibition of inflammatory, apoptosis, and regulation of actin cytoskeleton in podocytes effectively improve the DN by repairing the podocyte (8).

Eicosapentaenoic Acid (EPA) is a major component of fatty acids that has been studied to prevent and treat diabetic kidney disease $(9,10)$; however, the underlying mechanism remains obscure in DN. There is only one research indicating that the fatty acid induces apoptosis in podocytes, whereas $\omega-3$ polyunsaturated FFA reduces it (11). In current study, we also evaluated the effects of EPA on podocytes injury under high glucose treatment. A previous investigation showed that $n-3$ polyunsaturated fatty acid inhibits the sterol regulatory element-binding protein-1 (SREBP-1) to reduce albuminuria and renal dysfunction in a type II diabetic mice model (12). Thus, we supposed that EPA might regulate podocyte injury mediated by SREBP-1 and subsequently molecular signaling.

\section{Materials and methods}

\section{Reagent and assay kit}

Eicosapentaenoic Acid, 10417-94-4, Sigma, Merck. Cell counting assay kit-8 (CCK-8), ; The following antibodies were used for the western blots: podocin (ab50339, 1:1000), synaptopodin (ab224491, 1:1000), Bax (ab32503), Bcl-2 (ab182858, 1:1000), cleaved-caspase 3 (ab2302, 1:1000), caspase3 (ab13847, 1:1000), 
SREBP1 (ab55993, 1:1000), MYD88 (ab2064, 1:1000), TLR4 (ab13556, 1:1000), GAPDH (ab181602, 1:1000), HRP-conjugated goat anti-rabbit IgG (ab205718, 1:10000); EPA was dissolved in $99 \%$ isopropanol to prepare mother liquor, and diluted into serum free Dulbecco's modified Eagle's medium (DMEM; Gibco); CMH2DCFDA (General oxidative stress indicator), C6827, Invitrogen; Cell Apoptosis Kit with Annexin V Alexa Fluor 488 \& Propidium Iodide (PI), V13245, Invitrogen; Mouse IL-6 Quantikine ELISA kit, M6000B, R\&D systems; Mouse IL-1 beta/IL-1F2 Quantikine ELISA Kit, MLB00C, R\&D systems; Mouse TNF-alpha Quantikine Elisa Kit, MTA00B, R\&D systems.

\section{Cell culture}

The mouse podocyte cell line, MPC5 cells, was purchased from BioVector NTCC Inc. (China) and was culured in RPMI1640 medium (Gibco, Thermo Fisher Scientific) with $10 \%$ fetal bovine serum (FBS; Gibco, Thermo Fisher Scientific), $100 \mathrm{U} / \mathrm{ml}$ penicillin (Gibco, Thermo Fisher Scientific), $100 \mu \mathrm{g} / \mathrm{ml}$ streptomycin (Gibco, Thermo Fisher Scientific) and $50 \mathrm{U} / \mathrm{ml}$ interferon gamma (Gibco, Thermo Fisher Scientific) at $37{ }^{\circ} \mathrm{C}$. After the cells were incubated without interferon gamma for 14 days, the cells were differentiated. Cells were incubated with $25 \mathrm{mmol} / \mathrm{l}$ of high glucose (HG). We confirmed that the podocytes were differentiated mature by expression of podocin and synaptopodin by western blots.

\section{CCK-8}

Cell viability was measured using the CCK-8 assay. Cells were seeded in $5 \times 10^{3}$ cells/well in 96-well plate. After treatment, $100 \mu \mathrm{l}$ per well detection solution was incubated with cells at $37^{\circ} \mathrm{C}$ for $4 \mathrm{~h}$. The Varioskan Lux multi-function microplate instrument (Thermo Fisher Scientific) was used to detect the absorbance value at $450 \mathrm{~nm}$.

\section{Western blots analysis}

The proteins from the treated cells were extracted by PrepSEQ lysis buffer (Applied Biosystems). The samples were seperated by gel electrophoresis using 10 $\%$ Sodium dodecyl sulfate-polyacrylamide. Subsequently, the gels were transferred to PVDF membranes. $5 \%(\mathrm{~m} / \mathrm{v}$, in TBST) nonfat milk was used to block the membranes for $2 \mathrm{~h}$. Primary antibodies were incubated at $4{ }^{\circ} \mathrm{C}$ overnight and subsequently incubated with HRP-conjugated anti-rabbit antibodies at room temperature for $2 \mathrm{~h}$. The immuno-blots were detected by ECL plus Western Bloting substrate (Pierce, Thermo Fisher Scientific).

\section{Cell apoptosis analysis}

Treated cells were collected and centrifuged at $1000 \mathrm{~g}$ for $5 \mathrm{~min}$ at $4{ }^{\circ} \mathrm{C}$. The apoptotic cells were assessed by using Cell Apoptosis Kit with Annexin V Alexa Fluor 488 \& Propidium Iodide (PI) according to manual instructions. FACScan flow cytometer (Becton) was used to measure the cell apoptosis.

\section{Reactive oxygen species (ROS) analysis}

The alteration of ROS levels was assessed by using CM-H2DCFDA probe. Cells were plated with concentration of $5 \times 10^{3}$ cells/ well in 96-well plates. The cell culture medium was incubated with the probe for $30 \mathrm{~min}$ at $37^{\circ} \mathrm{C}$. Fluorescence was evaluated on a Varioskan Lux multi-function microplate instrument.

\section{Inflammatory factors analysis}

After cells treatment, the cells were lysed by ultrasonic fragmentation. The supernatant was determined by ELISA assay kit according to manufacture's instructions.
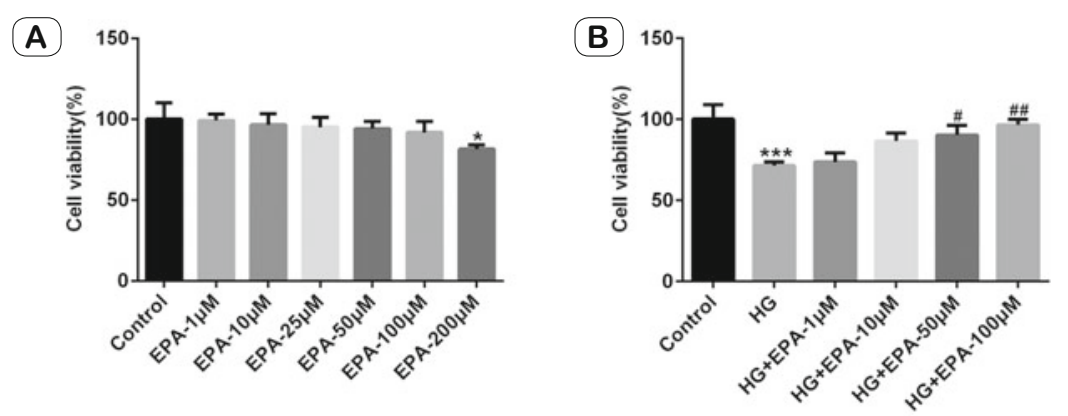

(C)
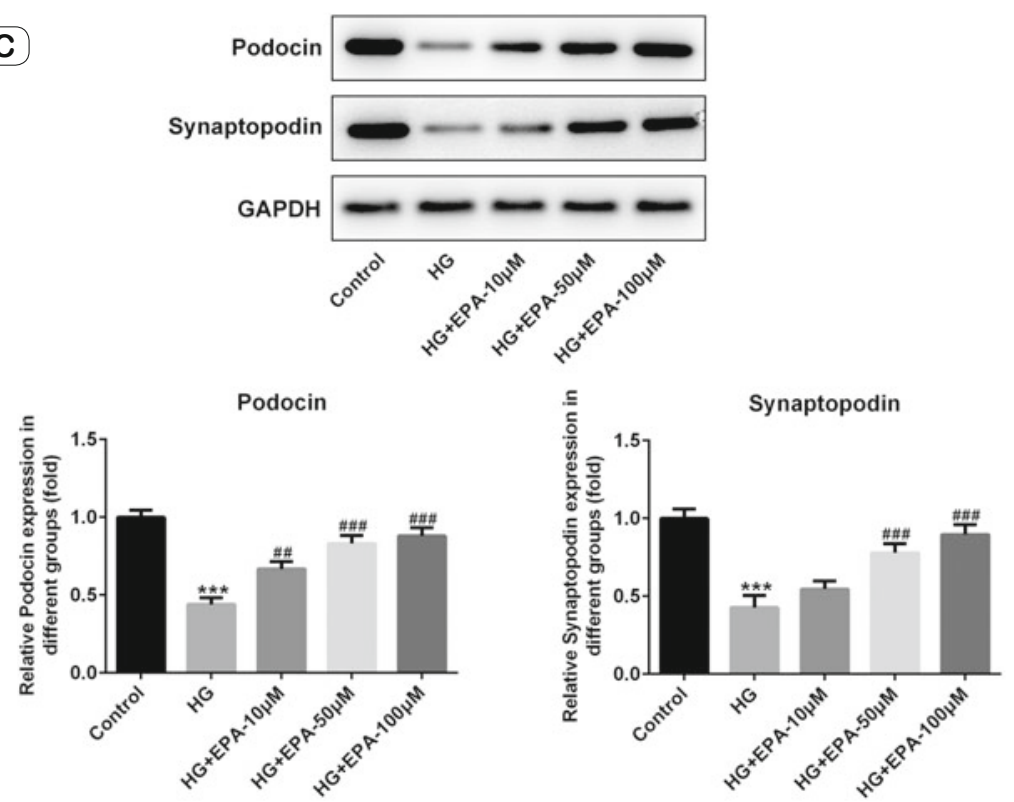

Fig. 1. EPA regulated podocytes dysfunction. (A) Differentiated MPC5 cells were treated with EPA $(1,10,25,50,100,200 \mu \mathrm{M})$ for $48 \mathrm{~h}(* \mathrm{p}<0.05$ vs control). The cell viability was detected by CCK-8. (B) Differentiated MPC5 cells were co-treated with high glucose $(25 \mathrm{mmol} / \mathrm{l}$, DMEM) and EPA $(1,10,50,100 \mu \mathrm{M})$ and cell viability was determined by CCK-8 $(* * * p<$ 0.001 vs control; \# $p<0.05$, \#\# $\mathrm{p}<0.01$ vs HG). (C) Differentiated MPC5 cells were treated with high glucose and EPA $(1,50,100 \mu \mathrm{M})$. The expression of podocin and synaptopodin was evaluated by western blots (*** $p<0.001$ vs control; \#\# $p<0.01$, \#\# $\mathbf{p}<0.001$ vs HG). 
$822-829$

(A)
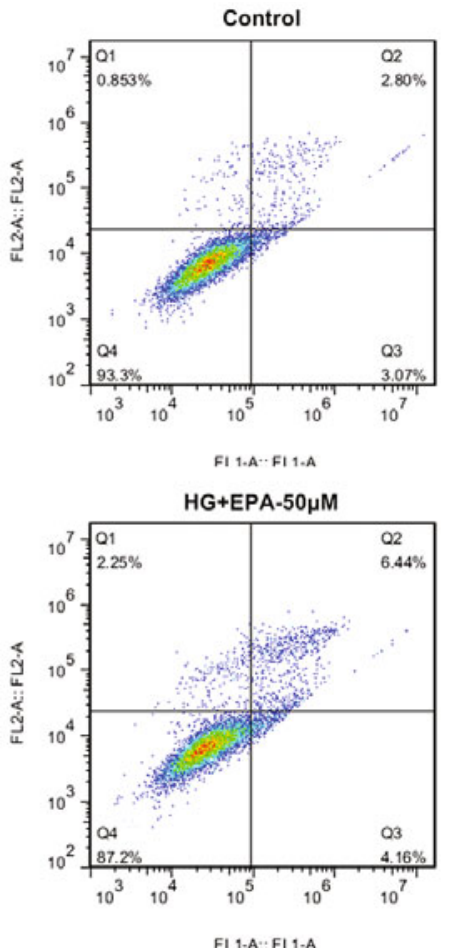

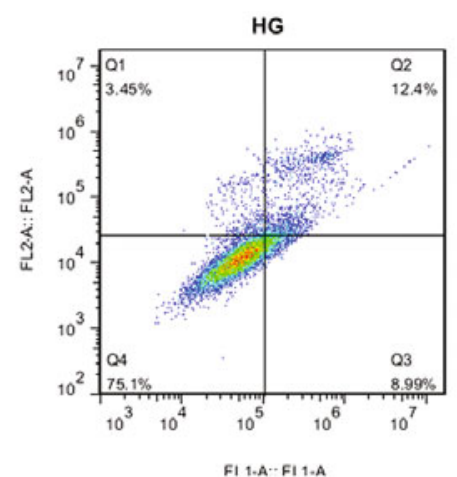

FI 1-A-Fi 1-A

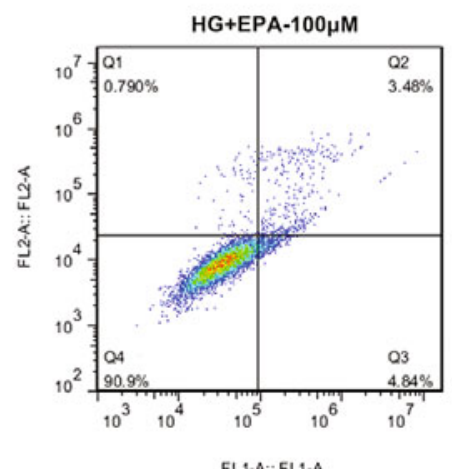

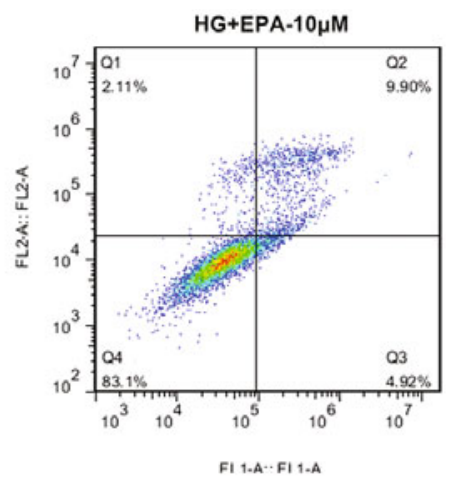

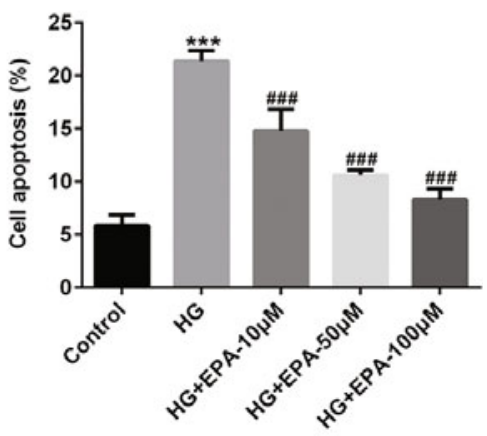

(B)
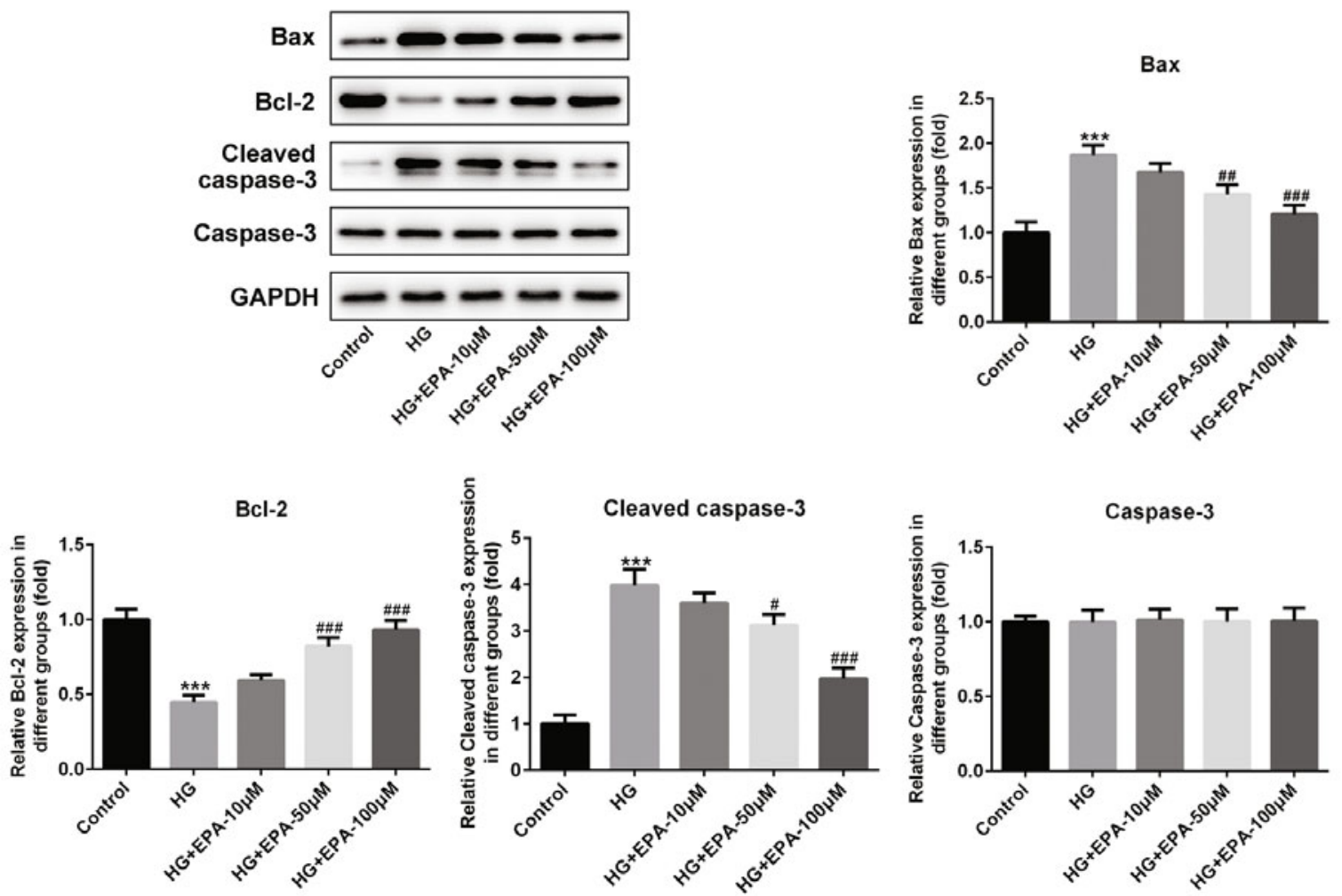

Fig. 2. EPA impeded high glucose induced apoptosis. Differentiated MPC5 cells were treated with high glucose (25 mmol/l, DMEM) or EPA $(10,50,100 \mu \mathrm{M})$ for $48 \mathrm{~h}$. (A) Flow cytometry was used to investigate the apoptosis. (B) The expression of Bax, Bcl-2, cleaved-caspase 3 and caspase 3 was detected by western blots. *** $p<0.001$ vs control; \# $p<0.05$, \#\#\# $\mathbf{p}<0.001$ vs HG. 

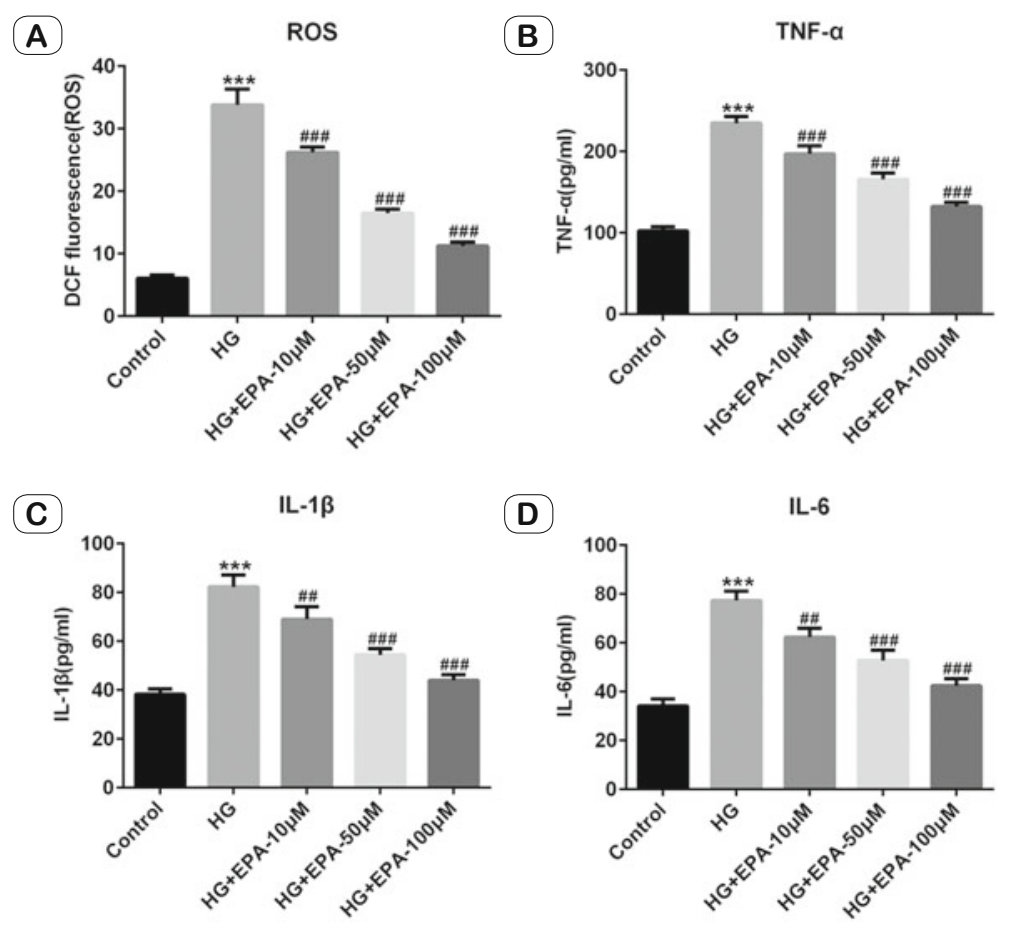

Fig. 3. EPA reduced the generation of ROS and pro-inflammatory factors. Differentiated MPC5 cells were treated with high glucose $(25 \mathrm{mmol} / \mathrm{l}, \mathrm{DMEM})$ or $\mathrm{EPA}(10,50,100 \mu \mathrm{M})$ for 48 h. (A) The generation of ROS was detected by CM-H2DCFDA in different groups. The production of TNF- $\alpha(B)$, IL-1 $\beta$ (C), and IL-6 (D) was regulated by ELISA assay kit. *** $p<$ 0.001 vs control; \#\# $\mathrm{p}<0.01$, \#\# $\mathrm{p}<0.001$ vs HG.

\section{Statistical analysis}

Data are presented as mean $\pm \mathrm{SD}$. The differences in present experiments were analyzed by using one-way ANOVA, followed by Tukey's multiple test among groups. A p value less than 0.05 was considered as significant difference.

\section{Results}

EPA improves podocytes function destroyed by high glucose.

We first evaluated the cytotoxicity of EPA on MPC5 cells, and found that EPA does not inhibit cell viability until treated with over $200 \mu \mathrm{M}$ (Fig. 1A). Subsequently, the results showed that EPA dramatically reversed the low cell viablity induced by high glucose (Fig. 1B). To explore the dysfunction of the podocytes, we used podocin and synaptopodin as a marker to study its regulation in podocytes. Management with high glucose in podocytes decreased the expression of podocin and synaptopodin, which was prevented by EPA therapy (Fig. 1C).

\section{EPA reduced high glucose induced apoptosis}

Further, results showed that EPA reduced the high glucose induced cell death by alleviating the cell apoptosis (Fig. 2A). Of the EPA, high glucose induced apoptosis, as was assessed by Bax-2, cleaved-caspase 3 , and Bcl-2, after $48 \mathrm{~h}$ of stimulation in a concentration dependent manner up to $100 \mu \mathrm{M}$ (Fig. 2B). EPA prominently reduced the expression of pro-apoptotic proteins including Bax-2 and cleaved-caspase 3 , and enhanced antiapoptotic protein $\mathrm{Bcl}-2$ expression.

EPA reduced ROS generation and inflammation in high glucose-treated podocytes

In cultured MPC5 cells, high glucose incresed the production of ROS, as evidenced by using a CM-H2DCFDA probe, whereas EPA treatment dramatically hindered it (Fig. 3A). Furthermore, EPA treatment inhibited the high glucose induced expression of TNF- $\alpha$, IL-1 $\beta$, and IL-6 (Fig. 3B-3D). EPA regulates the SREBP1/TLR4/ MYD88 signaling in high glucose-treated podocytes. Recent reports show that SREBP1 participates in the TLR4-mediated inflammation (13). EPA inhibited SREBP1 expression in high glucose treated MPC5 cells (Fig. 4A). Also, high glucose treatment enhanced the TLR4 and downstream MYD88 expression, and EPA inhibited the high glucose induced upregulaion of TLR4 and MYD88 (Fig. 4A). To further study the role of SREBP1, SREBP1 was overexpressed in MPC5 cells (Fig. 4B). Inbition of TLR4/MYD88 by EPA was activated by overexpression of SREBP1 (Fig. 5A). Overexpression of SREBP1 inhibited the effect of EPA on cell viability increased by high glucose (Fig. 5B). Moreover, SREBP1 upregulation reduced the podocin and synaptopodin expression in high glucose treated cells (Fig. 5C-5D). Upregulation of SREBP1 inhibited the EPA effects on apoptosis and expression of Bax, cleaved-caspase 3 and Bcl-2 (Fig. 6). In addition, EPA inhibited the high glucose induced ROS generation, secretion of TNF- $\alpha$, IL- $1 \beta$, and IL- 6 , which was inhibited by overexpression of SREBP1 (Fig. 7).

\section{Discussion}

The main findings of the current study are that there is a dramatical increase in apoptosis, inflammation and oxidative stress in the podocytes induced by high glucose, which was suppressed by treatment of EPA and mediated by SREBP1/TLR4/MYD88 signaling. Recently, dysfunction of podocytes is identified as the pathogenesis of DN, might participate in the early stages of this disease $(14,15)$. The cytoskeletal protein (synaptopodin) and the slit diaphragm (podocin) are performed to the functional and structural integrity of podocytes (16). High glucose-induced low expression of podocin and synaptopodin was dramatically enhanced by EPA administration, which may indicate that EPA can affect the function of podocyte.

EPA decreased mitochondrial-mediated apoptosis in podocytes in in vitro model. During DN, mitochondrial functions in podocytes are usually destroyed and principally the production of ROSis promoted (17). Some researches indicated that elevation 

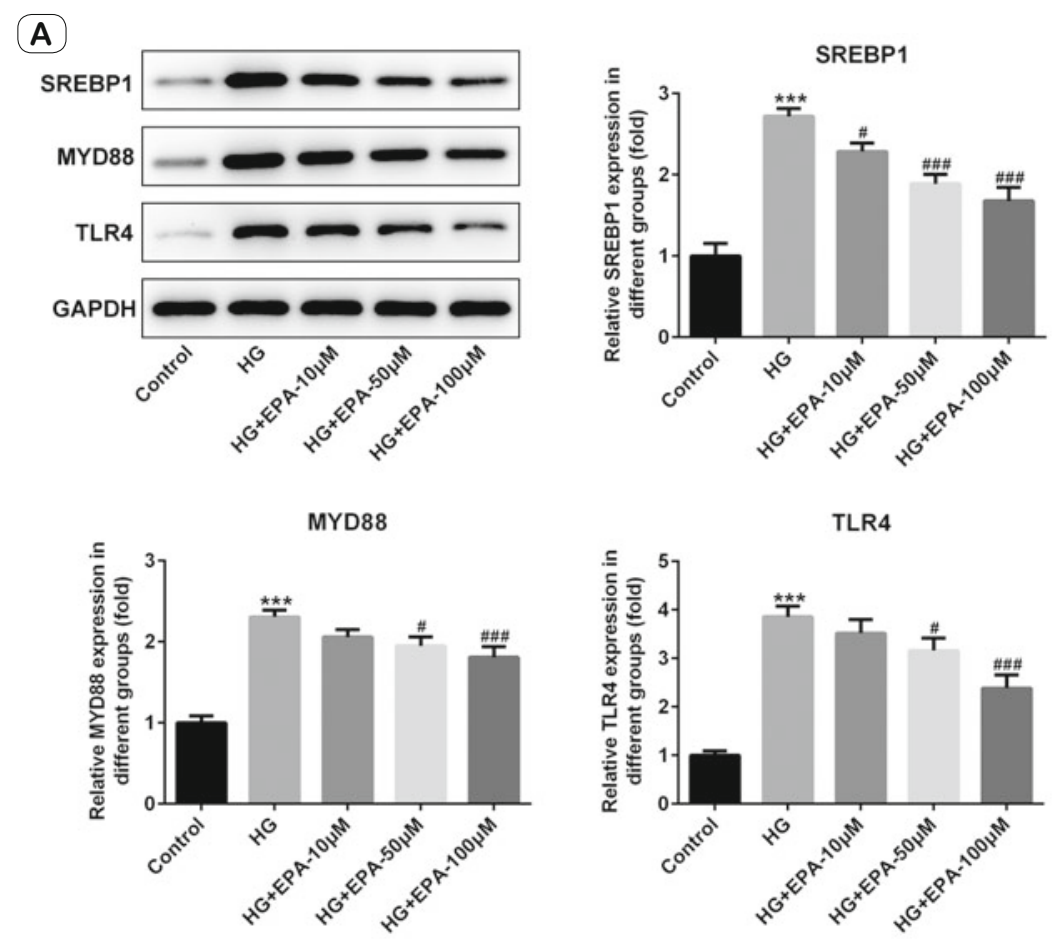

(B)

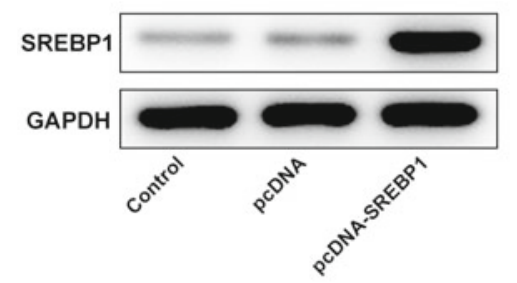

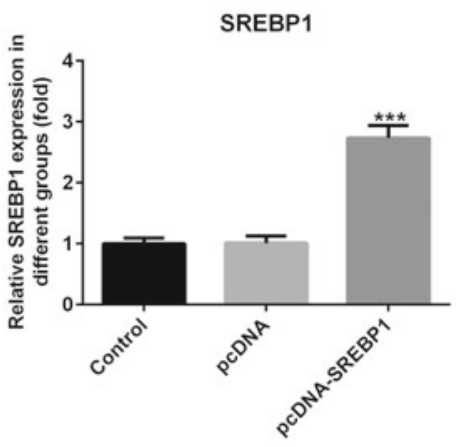

Fig. 4. EPA regulated SREBP1/TLR4/MYD88 signaling in high glucose treated podocytes. (A) The expression of SREBP1, TLR4, and MYD88 was detected by western blots in different groups. (B) The efficacy of transfection of overexpression of SREBP1 was evaluated by western blots. *** $\mathbf{p}<\mathbf{0 . 0 0 1}$ vs control; \# $\mathbf{p}<\mathbf{0 . 0 5}$, \#\# $\mathbf{p}<\mathbf{0 . 0 0 1}$ vs HG.

of ROS inhibited cell apoptosis by suppressing the expression of Bcl-2, a anti-apoptotic biomarker $(18,19)$. In the present study, we also found that ROS production is accompanied with loss of Bcl-2 and an increase of cleaved-caspase 3 and Bax induced by high glucose in podocytes and their alteration reversed by EPA. The data revealed that EPA might regulate the mitochondria mediated apoptosis in high glucose treated podocytes.

In the present investigation, we found for the first time that EPA inhibited the expression of SREBP-1, thereby reducing apoptosis and inflammation mediated by SREBP-1 in high glucosetreated podocytes. SREBP-1 has been reported to correlate with some renal diseases, such as glomerular fibrosis, chronic renal failure, and diabetic nephropathy (20-22). N-3 polyunsaturated fatty acid reduced albuminuria and renal dysfunction in a type II diabetic mice model by inhibiting SREBP-1 (12). In addition, we demonstrated that EPA hindered the TLR4/ MYD88 signaling evoked by high glucose, the effects of which could be reversed by overexpression of SREBP-1. TLR4 knockout increased the podocytes and podocin expression in renal tissues of the mice with STZ administration. TLR4/MYD88 signaling has been well understood in regulation of inflammation ${ }^{24}$. Similarly, inhibition of SREBP-1 by EPA also inhibited the expression of podocin and TLR4, subsequently reduced apoptosis and inflammation in high glucose-treated podocytes, indicating that EPA inhibited the podocytes dysfunction by regulation of SREBP-1.

\section{Conclusion}

EPA ameliorates the high glucose induced apoptosis and inflammation in podocytes by suppressing SREBP-1. Therefore, the protective role of EPA on diabetic nephropathy might be mediated by regulation of podocytes dysfunction. This basic research provides a strong theoretical basis for clinical research.

\section{References}

1. Alicic RZ, Rooney MT, Tuttle KR. Diabetic Kidney Disease: Challenges, Progress, and Possibilities. Clin J Am Soc Nephrol 2017; 12 (12): 2032-2045. DOI: 10.2215/CJN.11491116.

2. MacIsaac RJ, Ekinci EI. Progression of Diabetic Kidney Disease in the Absence of Albuminuria. Diabetes Care 2019; 42 (10): 1842-1844. DOI: $10.2337 /$ dci19-0030.

3. Heerspink HJL, Parving HH, Andress DL et al. Atrasentan and renal events in patients with type 2 diabetes and chronic kidney disease (SONAR): a double-blind, randomised, placebo-controlled trial. Lancet 2019; 393 (10184): 1937-1947. DOI: 10.1016/S0140-6736 (19)30772-X.

4. Menon MC, Chuang PY, He CJ. The glomerular filtration barrier: components and crosstalk. Int J Nephrol 2012; 2012: 749010. DOI: 10.1155/2012/749010.

5. Eftekhari A, Vahed SZ, Kavetskyy T et al. Cell junction proteins: Crossing the glomerular filtration barrier in diabetic nephropathy. Int J Biol Macromol 2020; 148: 475-482. DOI: 10.1016/j.ijbiomac. 2020.01.168.

6. Lemley KV. Diabetes and chronic kidney disease: lessons from the Pima Indians. Pediatr Nephrol 2008; 23 (11): 1933-1940. DOI: 10.1007/ s00467-008-0763-8. 
A
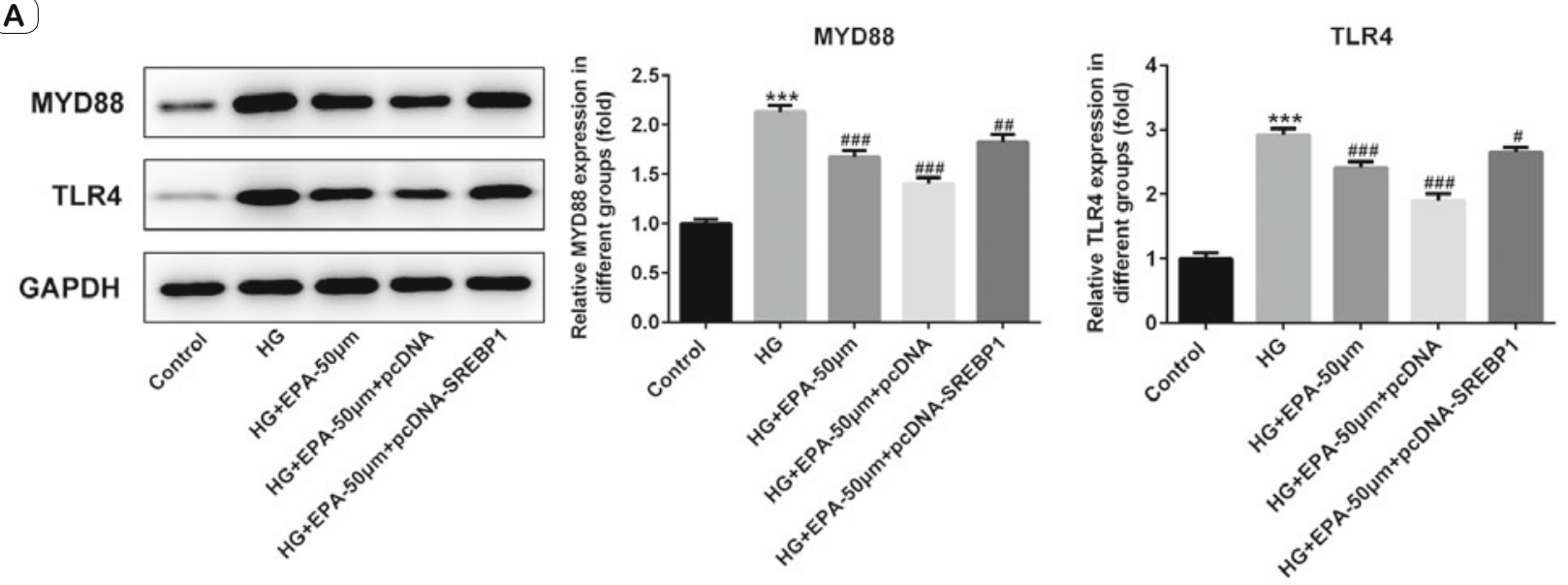

(B)

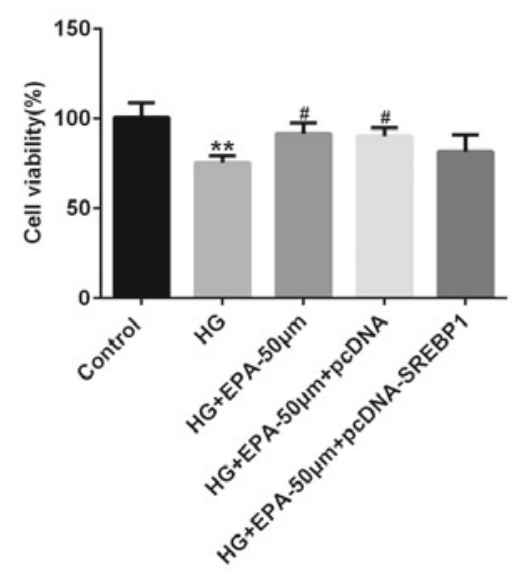

(C)

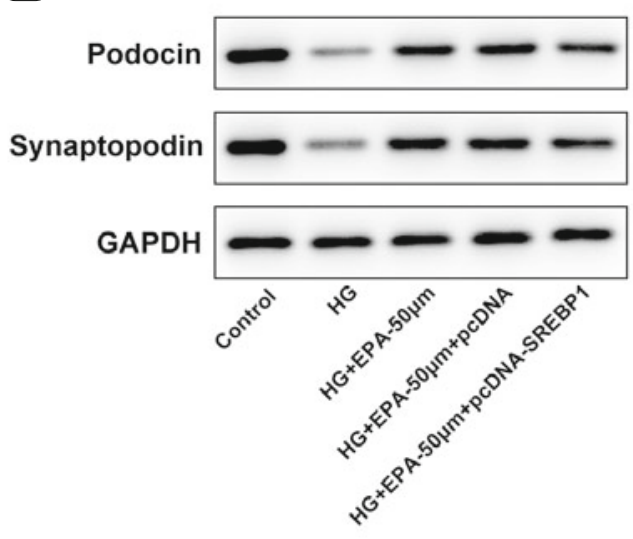

(D)
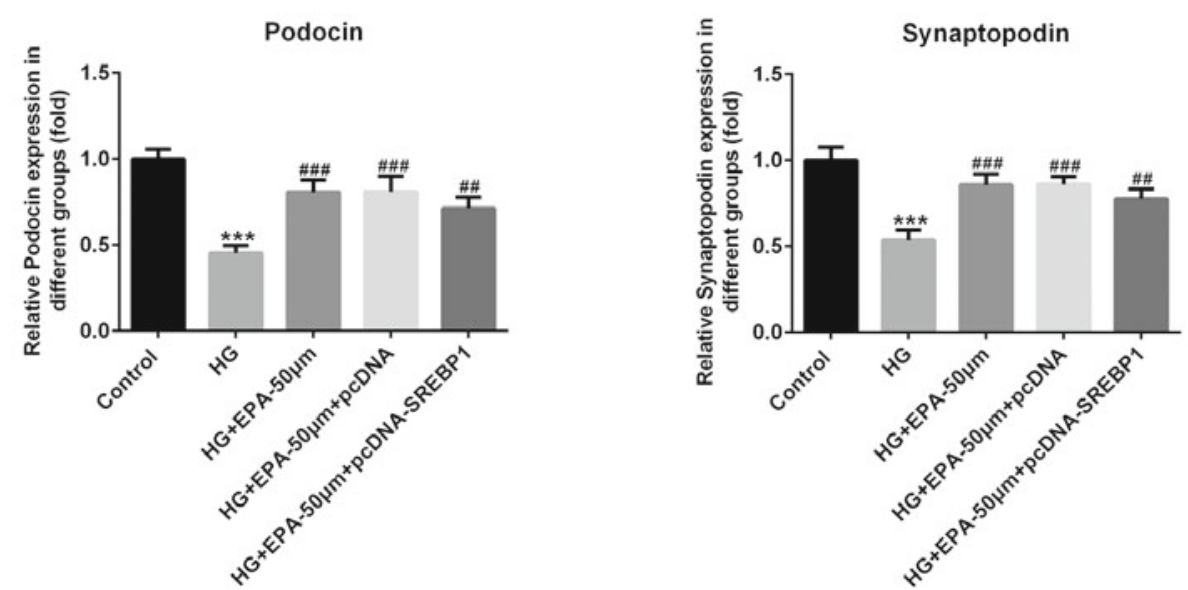

Fig. 5. Overexpression of SREBP1 prevents EPA effects on podocytes cell structure in high glucose treated podocytes. SREBP1 was overexpressed in high glucose treated podocytes with EPA treatment. (A) The expression of SREBP1, TLR4, and MYD88 was detected by western blots in different groups. (B) The cell viability was detected by CCK-8 in different groups. (C-D) The expression of podocin and synaptopodin was evaluated by western blots. ** $\mathrm{p}<0.01$, *** $\mathrm{p}<0.001$ vs control; \# $\mathrm{p}<0.05$, \#\# $\mathrm{p}<0.01$, \#\# $\mathrm{p}<0.001$ vs HG. 
$822-829$

(A)

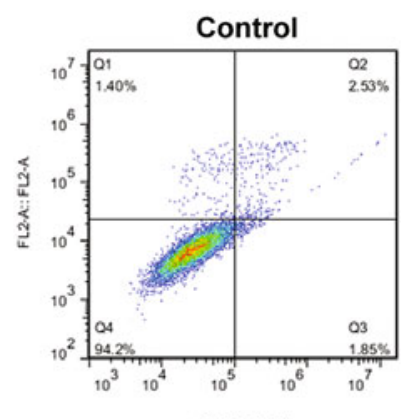

HG+EPA-50 $\mu \mathrm{m}+\mathrm{pcDNA}$

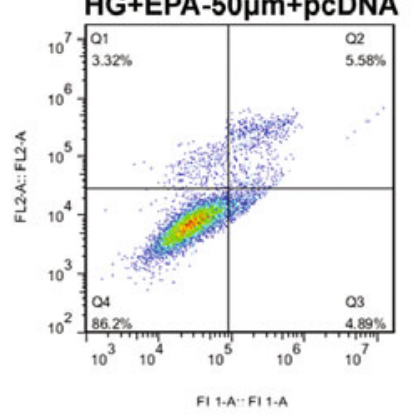

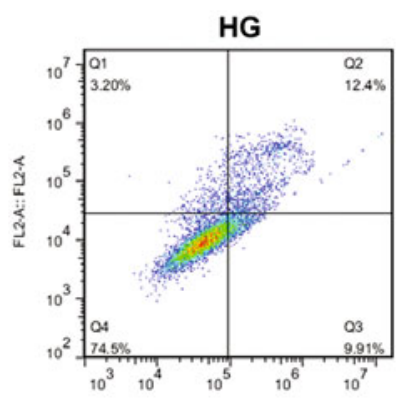

FI 1.A. F I1.A

\section{HG+EPA-50 $\mu m+p c D N A-S R E B P 1$}
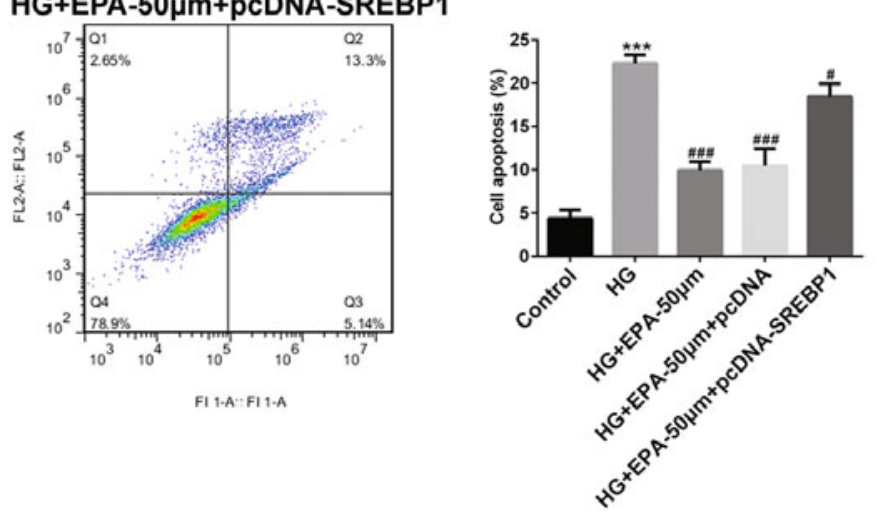

(B)
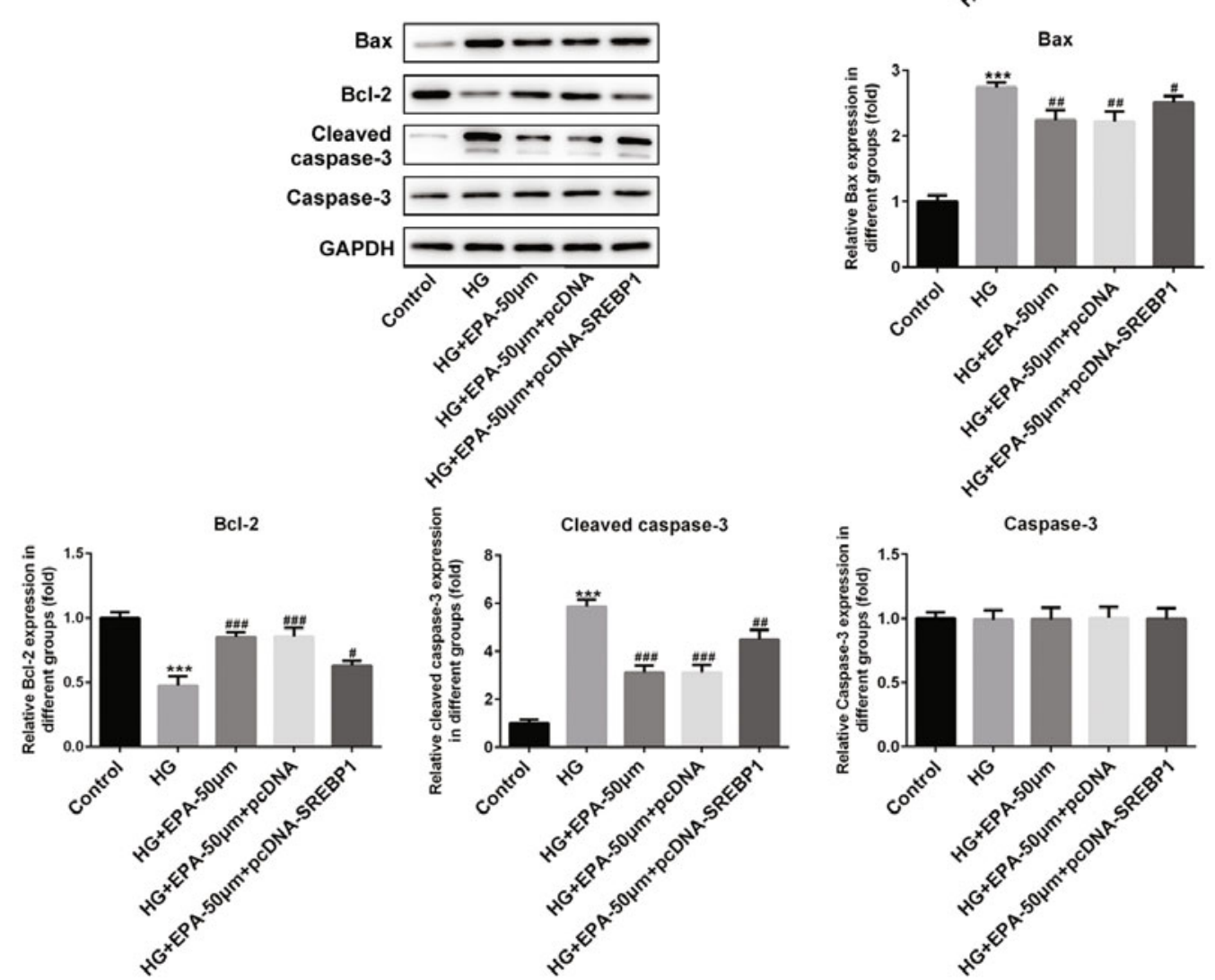

Fig. 6. Overexpression of SREBP1 re-enhanced the apoptosis reduced by EPA in high glucose treated podocytes. (A) Flow cytometry was used to investigate the apoptosis. (B) The expression of Bax, Bcl-2, cleaved-caspase 3 and caspase 3 was detected by western blots. $* * * \mathrm{p}<0.001$ vs control; \# $\mathrm{p}<0.05$, \#\# $\mathrm{p}<0.01$, \#\# $\mathrm{p}<0.001$ vs HG. 
A

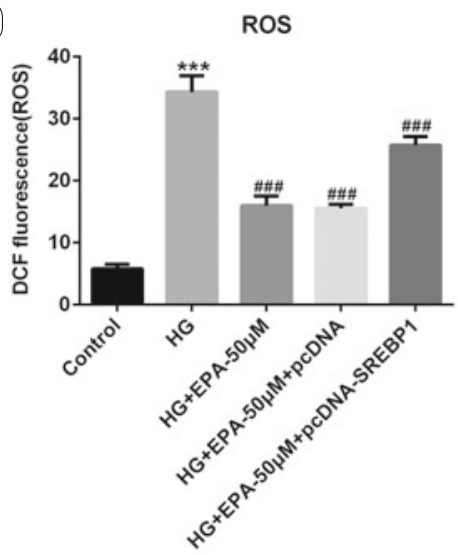

C

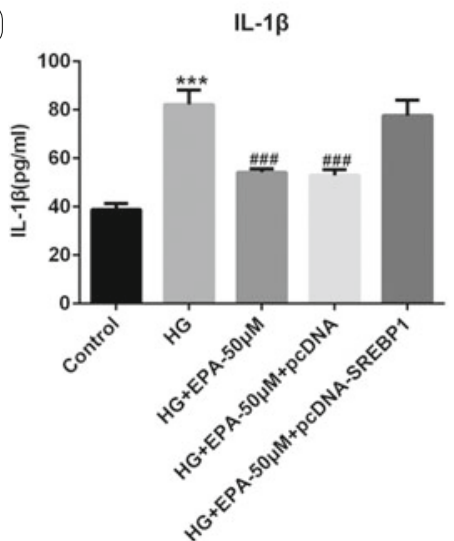

B

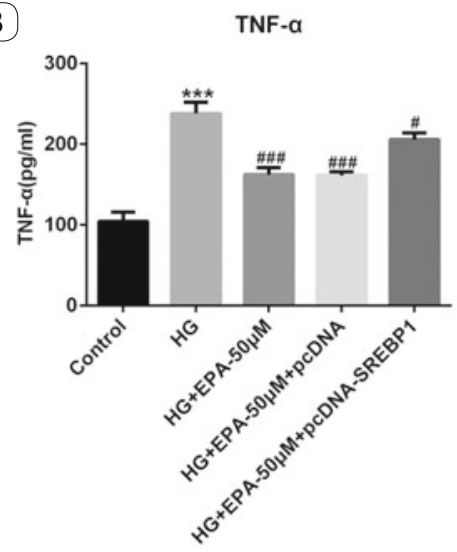

(D)

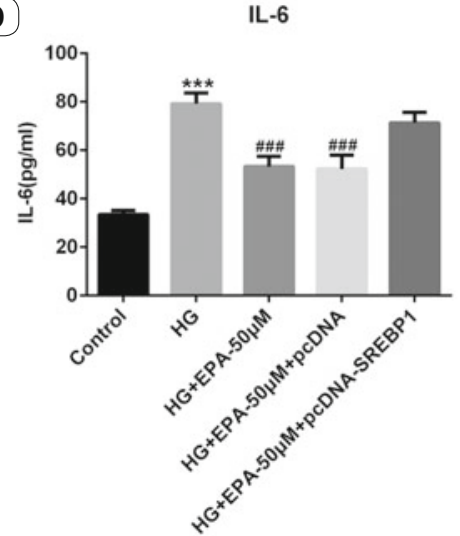

Fig. 7. EPA inhibits high glucose induced ROS generation and inflammation by alleviating SREBP1 expression. (A) The generation of ROS was detected by CM-H2DCFDA in different

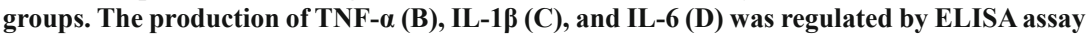
kit. ${ }^{* * *} p<0.001$ vs control; \# $p<0.05$, \#\#\# $p<0.001$ vs HG.

7. Liu W, Yang Y, Liu Y et al. Exogenous kallikrein protects against diabetic nephropathy. Kidney Int 2016; 90 (5): 1023-1036. DOI: 10.1016/j. kint.2016.06.018.

8. Liu M, Liang K, Zhen J et al. Sirt6 deficiency exacerbates podocyte injury and proteinuria through targeting Notch signaling. Nat Commun 2017; 8 (1): 413. DOI: 10.1038/s41467-017-00498-4.

9. Lee CC, Sharp SJ, Wexler DJ et al. Dietary intake of eicosapentaenoic and docosahexaenoic acid and diabetic nephropathy: cohort analysis of the diabetes control and complications trial. Diabetes Care 2010; 33 (7): 1454-6. DOI: $10.2337 / \mathrm{dc} 09-2245$.

10. Zhang M, Hagiwara S, Matsumoto $M$ et al. Effects of eicosapentaenoic acid on the early stage of type 2 diabetic nephropathy in KKA (y)/ Ta mice: involvement of anti-inflammation and antioxidative stress. Metabolism 2006; 55 (12): 1590-1598. DOI: 10.1016/j.metabol.2006.07.019.

11. Yasuda M, Tanaka Y, Kume S et al. Fatty acids are novel nutrient factors to regulate mTORC1 lysosomal localization and apoptosis in podocytes. Biochim Biophys Acta 2014; 1842 (7): 1097-1108. DOI: 10.1016/j. bbadis.2014.04.001.

12. Chin HJ, Fu YY, Ahn JM et al. Omacor, n-3 polyunsaturated fatty acid, attenuated albuminuria and renal dysfunction with decrease of SREBP-1 expression and triglyceride amount in the kidney of type II diabetic animals. Nephrol Dial Transplant 2010; 25 (5): 1450-1457. DOI: 10.1093/ndt/gfp695.
13. Oishi Y, Spann NJ, Link VM et al. SREBP1 Contributes to Resolution of Pro-inflammatory TLR4 Signaling by Reprogramming Fatty Acid Metabolism. Cell Metab 2017; 25 (2): 412-427. DOI: 10.1016/j.cmet.2016.11.009.

14. Maestroni S, Zerbini G. Glomerular endothelial cells versus podocytes as the cellular target in diabetic nephropathy. Acta Diabetol 2018; 55 (11): 1105-1111. DOI: $10.1007 / \mathrm{s} 00592-018$ 1211-2.

15. Zhong Y, Lee K, Deng Y et al. Arctigenin attenuates diabetic kidney disease through the activation of PP2A in podocytes. Nat Commun 2019; 10 (1): 4523. DOI: 10.1038/s41467-01912433-w.

16. Ornellas FM, Ramalho RJ, Fanelli $\mathrm{C}$ et al. Mesenchymal Stromal Cells Induce Podocyte Protection in the Puromycin Injury Model. Sci Rep 2019; 9 (1): 19604. DOI: 10.1038/s41598019-55284-7.

17. Fan Y, Yang Q, Yang Y et al. Sirt6 Suppresses High Glucose-Induced Mitochondrial Dysfunction and Apoptosis in Podocytes through AMPK Activation. Int J Biol Sci 2019; 15 (3): 701-713. DOI: 10.7150/ijbs.29323.

18. Hildeman DA, Mitchell T, Aronow B et al. Control of Bcl-2 expression by reactive oxygen species. Proc Natl Acad Sci U S A 2003; 100 (25): 15035-15040. DOI: 10.1073/pnas.1936213100.

19. Li D, Ueta E, Kimura $T$ et al. Reactive oxygen species (ROS) control the expression of Bcl-2 family proteins by regulating their phosphorylation and ubiquitination. Cancer Sci 2004; 95 (8): 644-650. DOI: 10.1111/j.1349-7006.2004. tb03323.x

20. Wang TN, Chen X, Li R et al. SREBP-1 Mediates Angiotensin II-Induced TGF-beta1 Upregulation and Glomerular Fibrosis. J Am Soc Nephrol 2015; 26 (8): 1839-1854. DOI: 10.1681/ASN.2013121332.

21. Korczynska J, Stelmanska E, Nogalska A et al. Upregulation of lipogenic enzymes genes expression in white adipose tissue of rats with chronic renal failure is associated with higher level of sterol regulatory element binding protein-1. Metabolism 2004; 53 (8): 1060-1065. DOI: 10.1016/j.metabol.2004.02.015

22. Li HY, Oh YS, Choi JW et al. Blocking lysophosphatidic acid receptor 1 signaling inhibits diabetic nephropathy in db/db mice. Kidney Int 2017; 91 (6): 1362-1373. DOI: 10.1016/j.kint.2016.11.010

23. Jialal I, Major AM, Devaraj S. Global Toll-like receptor 4 knockout results in decreased renal inflammation, fibrosis and podocytopathy. J Diabetes Complications 2014; 28 (6): 755-761. DOI: 10.1016/j.jdiacomp.2014.07.003.

24. Lu S, Zhang H, Wei $X$ et al. 2-dodecyl-6-methoxycyclohexa-2,5diene-1,4-dione isolated from Averrhoa carambola L. root ameliorates diabetic nephropathy by inhibiting the TLR4/MyD88/NF-kappaB pathway. Diabetes Metab Syndr Obes 2019; 12:1355-1363. DOI: 10.2147/ DMSO.S209436.

Received March 31, 2020 Accepted May 19, 2020. 